\title{
Degradation of Instrumentation Amplifiers Due to the Nonionizing Energy Loss Damage
}

\author{
F. J. Franco, J. Lozano, J. P. Santos, and J. A. Agapito
}

\begin{abstract}
Tests on instrumentation amplifiers exposed to neutron radiation have been done. The tested devices were commercial instrumentation amplifiers or designed with rad-tol commercial operational amplifiers. The results show changes in frequency behavior, gain, offset voltage, output saturation voltages, and quiescent current. The radiation tolerance is bigger in amplifiers with JFET input stage or with large frequency bandwidth and is smaller if the amplifier has been designed for reducing the power consumption. The IAs built with OPAMPs have a higher tolerance than the commercial ones, but they have disadvantages: high temperature influence, low CMRR, etc.
\end{abstract}

Index Terms-COTS, displacement damage, instrumentation amplifiers, neutron tolerance, operational amplifiers.

\section{INTRODUCTION}

$\mathbf{T}$ HE electronic instrumentation that will be used in the large hadron collider (LHC) cryogenic system will receive a neutron radiation between $10^{13}-10^{14}$ particles $/ \mathrm{cm}^{2}$ and a gamma radiation dose in the order of several hundreds of Gy during ten years. The measure of the control sensors will require the use of instrumentation amplifiers. These amplifiers must guarantee to work with such radiation dose.

\section{A. The Radiation Inside the Collider}

The LHC that is being built nowadays at the European Organization for the Nuclear Research (CERN) needs the use of powerful electromagnets so that heavy particles flying near light velocity could turn and keep confined inside the accelerator ring. The electric current that flows through the magnets is very high, and the use of a NbTi superconducting alloy at $1.8 \mathrm{~K}$ is needed [1]. The collider ring must be covered with liquid helium to reach this temperature, and it is essential to know the He characteristics.

The correct functioning of the electronic control instrumentation must be guaranteed during the full collider lifetime. If the particles are charged, they will not be able to escape from the collider because of the magnetic field. The charged particle leakage will be important only at the intermediate gap between the magnets. On the other hand, if the particle is neutral, it will pierce the metallic armor that isolates the beam and arrive at the electronic instrumentation that measures the He parameters. Most of the escaped particles will be neutrons, although other neutral particles, such as pions, mesons, etc. can affect

This work was supported by the cooperation agreement K476/LHC between CERN and UCM and by the Spanish Research Agency CICYT (TIC98-0737) and in part by ITN.

The authors are with the Departmento de Física Aplicada III, Facultad de CC. Físicas, Universidad Complutense de Madrid, 28040 Madrid, Spain (email: monti@fis.ucm.es). the electronic system. Also, the gamma radiation produced by the particles can alter the measurement instrumentation. A 10-Gy/year dose is expected along the tunnel, but it will be increased up to $50 \mathrm{~Gy} / \mathrm{year}$ in the gap between magnetic dipoles due to the charged particle leakage. Definitively, the electronic instrumentation will receive a dose about $5 \cdot 10^{13}$ $\mathrm{n} \cdot \mathrm{cm}^{2}$ and several hundreds of Gy during ten years.

\section{B. About Instrumentation Amplifiers}

An instrumentation amplifier is a gain differential device ready to work in a hostile environment for accurate measures. These devices need very high input impedance, low bias and offset currents, and balanced inputs to minimize the common mode gain. Moreover, the internal parameters must be very stable to avoid the effect of temperature and power supplies variations [2].

The instrumentation amplifier gain is controlled with an external resistance that is calculated with a mathematical expression given by the manufacturer. The output stage is designed for grounded loads. There is an additional input called "Reference" that is joined to ground or to the load if it is far away from the measurement network. Two other inputs and the negative power supply can be connected by a potentiometer to remove the output offset voltage.

Generally, the design of instrumentation amplifiers is based on the operational amplifier properties. In spite of the fact that there are several circuits with a global behavior like the instrumentation amplifiers [3], [4], [5], the most used network is shown in Fig. $\mathbf{1}$ and is known as a "classic three opamp network." In this network, the gain is selected by an external resistor called $R_{G}$ and the value is

$$
O U T=R E F+\left(1+\frac{2 \cdot R}{R_{G}}\right) \cdot\left(V_{+}-V_{-}\right)
$$

This network can be found inside the tested integrated instrumentation amplifiers. The great advantage of this structure is the very large value of CMRR due to the symmetry ratio possibility since the number of basic devices is reduced [2].

However, in these networks, the CMRR reduction is obtained by building identical transistors, and in the classical network, only matched resistors are needed. Due to its simplicity, this topology is widely used instead of the other ones with less number of devices.

\section{Tested Instrumentation Amplifiers}

The search for rad-tol instrumentation amplifiers was done following two investigation lines. First, commercial instrumen- 


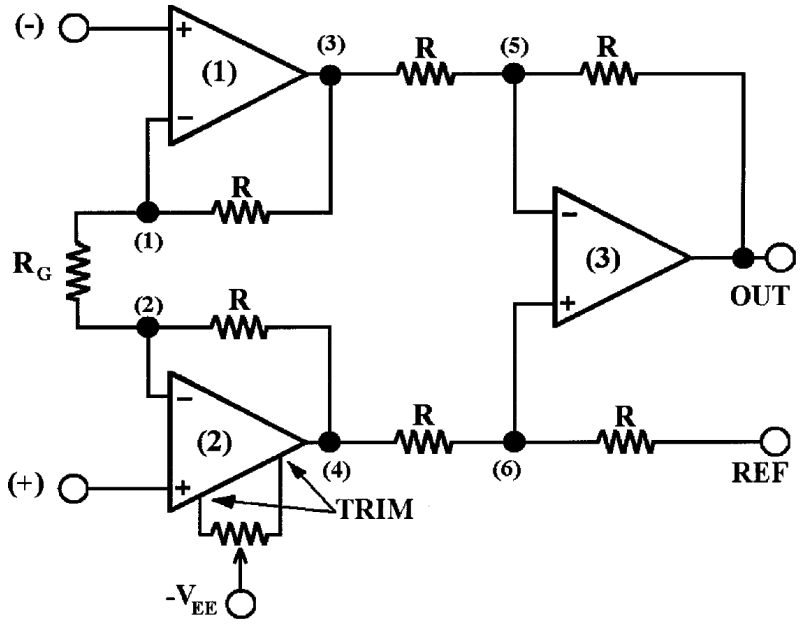

Figure 1. Classic three opamp network.

tation amplifiers from different manufacturers and technologies were tested. Second, the networks of the amplifiers were built with rad-tol operational amplifiers, $1 \%$ accuracy resistors, and potentiometers. These were used to decrease the offset voltage and increase CMRR.

The tested commercial instrumentation amplifiers are shown in Table I. The main characteristics are provided by the manufacturers. The standard values of some parameters, the device purpose, and the technology are shown in Table I. The internal design is based on the topology of Fig. 1, although some of them have been improved. The INA114, INA116, INA118, and INA121 amplifiers have an over voltage protection system, and the scheme can be found in the device datasheet. On the other hand, the INA116 amplifier has guards to insulate the inputs and its design is unknown.

OPA627AP and TLE2071CP operational amplifiers were used to build the instrumentation amplifier with discrete devices. They were chosen because they had shown a great tolerance to radiation [6]. Overall, $22-\mathrm{k} \Omega$ accurate resistors were used to assemble the network, although the resistor between the output and the node 5 were replaced by an $18-\mathrm{k} \Omega$ resistor and a $10-\mathrm{k} \Omega$ potentiometer. This potentiometer is used to tune the amplifier and increase the CMRR value. In order to trim the operational amplifier offset, other potentiometers were placed.

\section{Test Conditions}

\section{A. Description of Neutron Source}

The devices were irradiated in a thermally conditioned facility built at the Portuguese Research Reactor (ITN). A 0.7 $\mathrm{cm}$ thick boral shield removes the thermal neutron components of the beam emitted by the reactor core. The neutron energy spectrum is rather constant between $0.6-3.0 \mathrm{MeV}$, and the other components are negligible. A $5 \cdot 10^{13} \mathrm{n} / \mathrm{cm}^{2}$ fluence is reached after five days with $14 \mathrm{~h}$ of activity and 10 of standby. A 4-cm-thick lead shield reduces the gamma dose below $2 \mathrm{kGy}$ in the center of the cavity. The fission neutron fluence was measured with $\mathrm{Ni}$ detectors placed in the center of the boxes containing the test boards. They showed that the samples received a neutron fluence between $3.46 \cdot 10^{13}$ $\& 1.1 \cdot 10^{14} \mathrm{n} / \mathrm{cm}^{2}$. Integration dosimeters were placed on the first and last board and revealed that the gamma dose inside the cavity was between 1.3-2.3 kGy.t Also, an ionization chamber monitorized the total gamma dose in the center of the cavity and its value reached $1.6 \mathrm{kGy}$.

\section{B. Data Acquisition System}

During the irradiation, the amplifiers, both integrated and built, were assembled on PCBs with a $R_{G}=470 \Omega$, a gain of about 107, and biased with $\pm 15 \mathrm{~V}$ supplies. A minimum of three samples of each device were exposed. Every ten minutes, an input voltage sweep was applied to measure the values of gain, offset voltage, and bias currents. During the sweep standby period, mechanical relays insulated the amplifiers. A PC controlled system with a Keithley 236 current source, Keithley 7002 switch system, and Keithley $20029 \frac{1}{2}$ digit multimeter was built. The instruments were connected to the test boards with a $4 \mathrm{~m}$ shield pipe with a resistivity less than $0.075 \Omega \cdot m$. The instruments were controlled by a program developed in Testpoint.

Other magnitudes as those related to frequency bandwidth or supply could only be measured after the irradiation when the samples did not have any activity. The samples had to be isolated at room temperature for a month.

\section{RESULTS IN INTEGRATED AMPLIFIERS}

Several amplifiers were destroyed when they reached radiation doses much lower than the final value. Table II shows the highest dose that they could bear.

Table II

HIGHEST NEUTRON TOLERANCE OF INSTRUMENTATION AMPLIFIERS

\begin{tabular}{lccc}
\hline AD620AD & $10^{12} \mathrm{n} \cdot \mathrm{cm}^{-2}$ & INA116PA & $2 \cdot 10^{12} \mathrm{n} \cdot \mathrm{cm}^{-2}$ \\
\hline INA118PB & $3.6 \cdot 10^{12} \mathrm{n} \cdot \mathrm{cm}^{-2}$ & AD624AD & $2 \cdot 10^{13} \mathrm{n} \cdot \mathrm{cm}^{-2}$ \\
\hline INA121PA & $2 \cdot 10^{13} \mathrm{n} \cdot \mathrm{cm}^{-2}$ & INA114AP & $2.5 \cdot 10^{13} \mathrm{n} \cdot \mathrm{cm}^{-2}$ \\
\hline
\end{tabular}

These values are repeated on all the samples of each amplifier. Only a few disagreements were observed on the most separated samples from the reactor core. On these ones, the dose is slightly higher, although it can be attributed to the higher number of reactor standby periods where a fraction of defects were removed from the semiconductor and the lattice was partially regenerated.

The INA110KP and INA111PA were the most tolerant devices to neutron radiation damage. There is not a fixed neutron fluence that the amplifiers can receive without being destroyed, e.g., an INA110KP sample that received 1.1.10 14 $\mathrm{n} / \mathrm{cm}^{2}$ started to deteriorate with a $4.5 \cdot 10^{13} \mathrm{n} / \mathrm{cm}^{2}$ dose and was destroyed at $6.8 \cdot 10^{13} \mathrm{n} / \mathrm{cm}^{2}$. However, other samples received this dose without great damage. This can be related to the lattice recovery during the standby periods because the destroyed sample was the closest to the core. 
Table I

TESTED COMMERCIAL INSTRUMENTATION AMPLIFIERS

\begin{tabular}{|c|c|c|c|c|c|c|c|c|c|}
\hline Name & Manufact. & Technology & Purpose & Offset & Bias Curr. & $\begin{array}{c}\text { Freq }-3 \mathrm{~dB} \\
(\text { Gain }=100)\end{array}$ & Slew Rate & Quies. Curr. & Sh. Circ. \\
\hline $\mathrm{AD} 620 \mathrm{AN}$ & Analog & Bipolar & Low Power & $30 \mu \mathrm{V}$ & $0.5 \mathrm{nA}$ & $120 \mathrm{kHz}$ & $1.2 \mathrm{~V} / \mu \mathrm{s}$ & $0.9 \mathrm{~mA}$ & $18 \mathrm{~mA}$ \\
\hline ADDீ24AD & Analog & Bipolar & Precision & $200 \mu \mathrm{V}$ & An & $150 \mathrm{kHz}$ & $5.0 \mathrm{~V} / \mu \mathrm{s}$ & $3.5 \mathrm{~mA}$ & -- \\
\hline INA110KP & Burr-Brown & $\begin{array}{c}\text { Bipolar + } \\
\text { JFET Input }\end{array}$ & Fast Settling & $100 \mu \mathrm{V}$ & $20 \mathrm{pA}$ & $470 \mathrm{kHz}$ & $17 \mathrm{~V} / \mu \mathrm{s}$ & $3.0 \mathrm{~mA}$ & $25 \mathrm{~mA}$ \\
\hline INA111BP & Burr-Brown & $\begin{array}{c}\text { Bipolar + } \\
\text { JFET Input }\end{array}$ & High Speed & $100 \mu \mathrm{V}$ & $2 \mathrm{pA}$ & $450 \mathrm{kHz}$ & $17 \mathrm{~V} / \mu \mathrm{s}$ & $3.3 \mathrm{~mA}$ & $30 \mathrm{~mA}$ \\
\hline INA114AP & Burr-Brown & Bipolar & Precision & $10 \mu \mathrm{V}$ & $5 \mathrm{nA}$ & $10 \mathrm{kHz}$ & $0.6 \mathrm{~V} / \mu \mathrm{s}$ & $2.2 \mathrm{~mA}$ & $20 \mathrm{~mA}$ \\
\hline INA116P & Burr-Br & $\begin{array}{c}\text { Bipolar + } \\
\text { DiFET Input }\end{array}$ & $\begin{array}{c}\text { Very low } \\
\text { input current }\end{array}$ & $500 \mu \mathrm{V}$ & $3 \mathrm{fA}$ & $70 \mathrm{kHz}$ & $0.8 \mathrm{~V} / \mu \mathrm{s}$ & $1.0 \mathrm{~mA}$ & $5 \mathrm{~mA}$ \\
\hline INA118PB & Burr-Brown & Bipolar & $\begin{array}{l}\text { Precision }+ \\
\text { Low Power }\end{array}$ & $10 \mu \mathrm{V}$ & $1 \mathrm{nA}$ & $70 \mathrm{kHz}$ & $0.9 \mathrm{~V} / \mu \mathrm{s}$ & $0.35 \mathrm{~mA}$ & $5 \mathrm{~mA}$ \\
\hline INA121PA & Burr-Brown & $\begin{array}{c}\text { Bipolar + } \\
\text { JFET Input }\end{array}$ & Low Fower & $200 \mu \mathrm{V}$ & $4 \mathrm{pA}$ & $50 \mathrm{kHz}$ & $0.7 \mathrm{~V} / \mu \mathrm{s}$ & $0.45 \mathrm{~mA}$ & $14 \mathrm{~mA}$ \\
\hline
\end{tabular}

\section{A. Differential Gain}

The value of this parameter was measured every ten minutes, whereas the amplifiers were irradiated. The gain evolution has two phases: In the first moments of the irradiation, the gain remains stable or there is a slight decrease of the gain about $1 \%$ of the initial value (Figs. $2 \& 3$ ). When the fluence is about to reach the highest one that the amplifiers can tolerate, the shift of the gain increases. There are two typical behaviors: The least tolerant devices suffered a sudden gain drop to 0 (Figs. $2 \& 3$ ), but the INA110KP and INA111BP showed a soft decrease (Fig. 4). The INA114AP gain increases before the amplifiers were destroyed (Fig. 3).

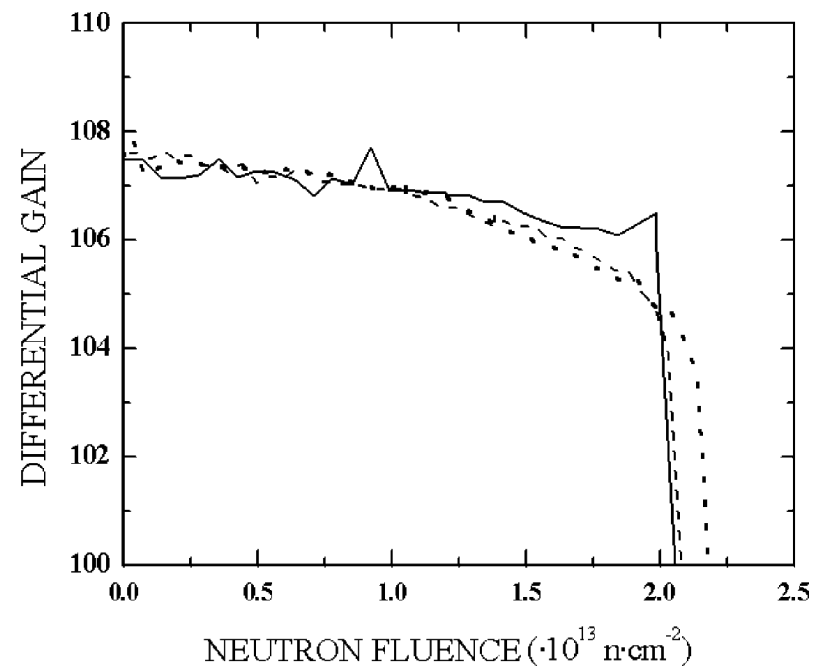

Figure 2. INA121PA gain versus neutrons.

\section{B. Input Offset Voltage}

Due to the fast destruction of the AD620AN, INA116PA, and INA118PB amplifiers, few values of the input offset

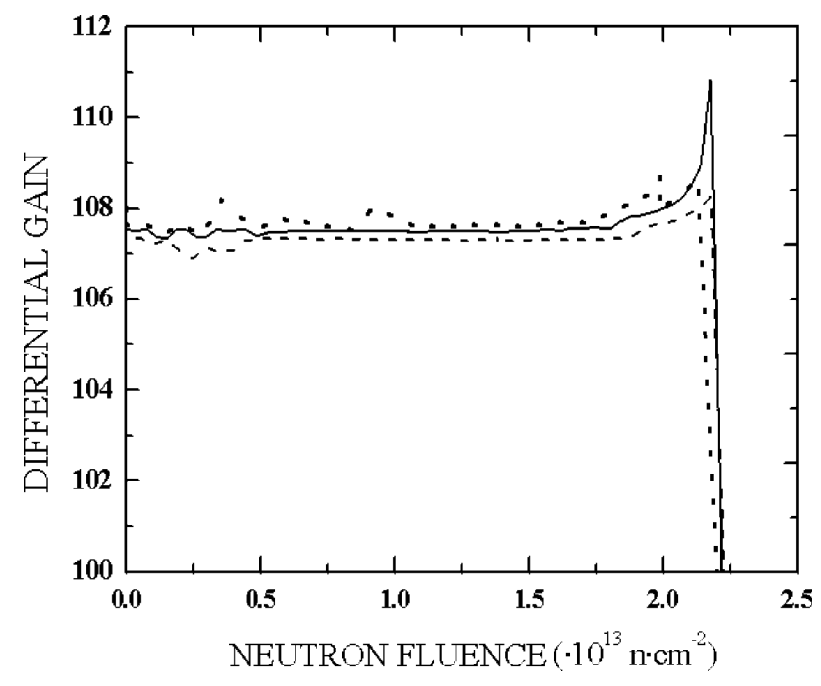

Figure 3. INA114AP gain versus neutron fluence.

voltage could be taken. A growth of several $\mathrm{mV}$ was observed before the final collapse.

The evolution of the input offset voltage on the more tolerant devices depended on the input stage technology. The models with a bipolar input (INA114AP, AD624AD) suffered a similar offset evolution on all samples (Fig. 5). On the other hand, the samples of the devices with JFET input stage (INA110KP, INA111BP, and INA121PA) show different shifts though they belonged to the same manufacturer batch (Fig. 6).

\section{Bias \& Offset currents}

On JFET input amplifiers, no considerable growth of the currents was observed. Only the INA121PA showed an increase of $-20 \mathrm{nA}$ during the exposition. The damage is greater on the amplifiers with bipolar input stage. The growth was hardly observed on AD620AN and INA118PB because they 


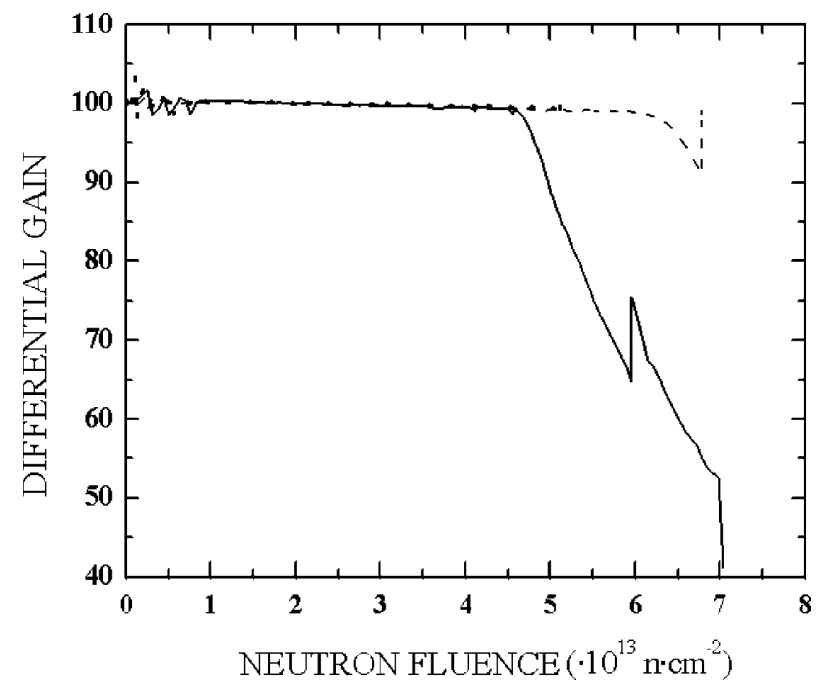

Figure 4. INA110KP gain versus neutron fluence.

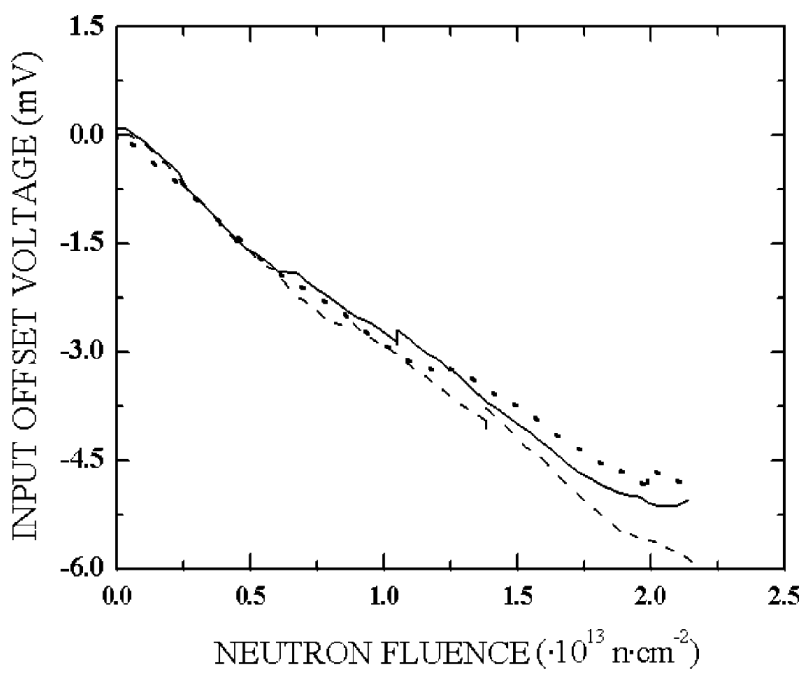

Figure 5. INA114AP input offset voltage versus neutron fluence.

were quickly destroyed, but the INA114AP amplifier reached a bias input current of $-15 \mathrm{nA}$, and for the AD624AD, the damage was even higher due to the fact that the bias current rose up to $150 \mathrm{nA}$.

\section{D. -3 dB Frequency and Slew Rate}

Although an INA111BP sample worked after the irradiation, the frequency behavior could not be inspected because the output signal with square or sinusoidal inputs was so distorted that the former parameters could not be taken. So, only the data from INA110KP are available. Fig. 7 shows the ratio between the final and initial values of the $-3 \mathrm{~dB}$ frequency, with $G=10$, $G=100$, and slew rate related to the total neutron fluence that the samples received. A worsening of frequency response is observed.

\section{E. Quiescent current}

The quiescent current is the current supplied by $+\mathrm{V}_{C C}$ if all inputs are grounded. We could measure this parameter

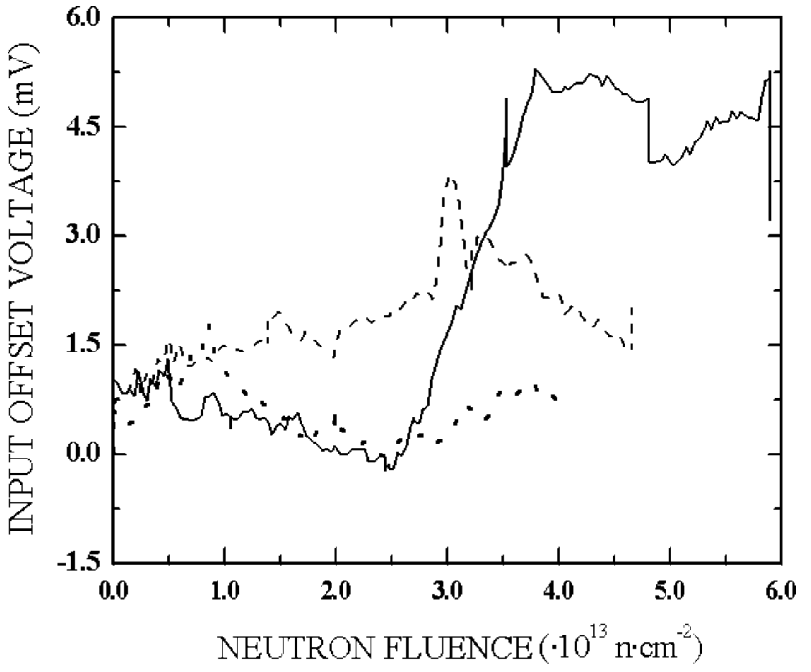

Figure 6. INA111BP input offset voltage versus neutrons.

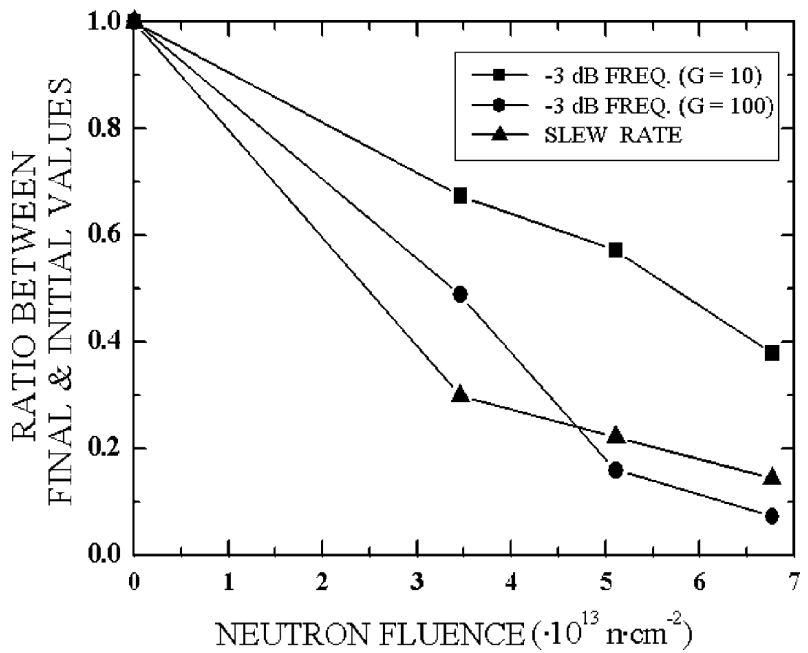

Figure 7. Degradation of the frequency response of the survived INA110KP amplifiers.

on all the amplifiers because it does not depend on their correct behavior. Three typical behaviors were found: For the AD620AN and INA118PB amplifiers, low-power bipolar and not rad-tol, a reduction of the current consumption until a $10-20 \%$ of the initial value takes place. The AD624AD, INA110KP, and INA111NP, which had a high initial quiescent current, suffer a decrease on this parameter, but the value does not depend on the total neutron fluence. Depending on the device, the current values are comprised between $60 \%-80 \%$ of the initial current.

The other amplifiers have an intermediate initial quiescent current, and there is clear dependence on the fluence. Fig. 8 shows the results obtained on the analyzed samples.

\section{F. Input-Output Function}

After the deactivation of the devices, the relationship between input-output of the surviving INA110KP and INA111BP at $\pm 15 \mathrm{~V}$ with a gain of 107 was measured in the full range of input voltage. A straight line with a slope $=107$, 


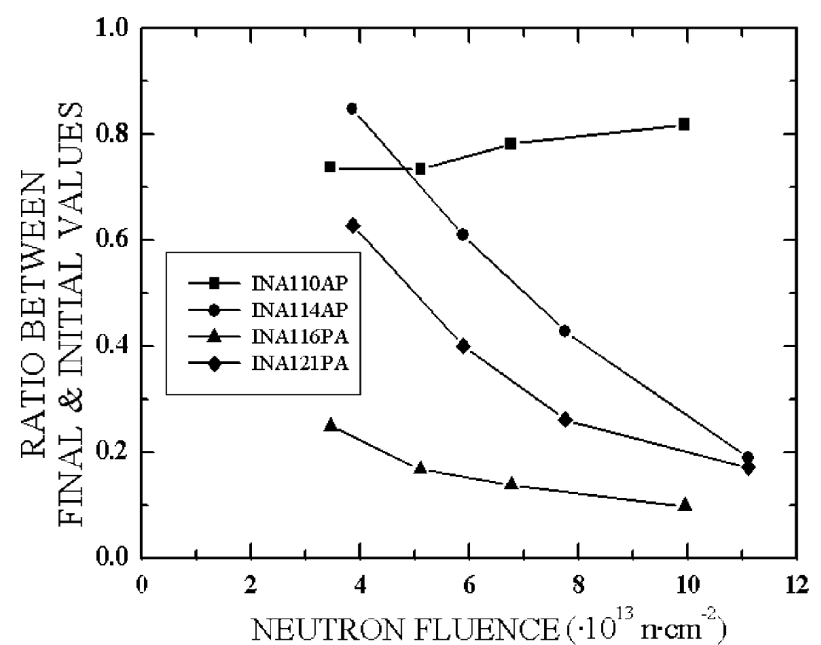

Figure 8. Evolution of quiescent current versus neutron fluence.

which is truncated when the output is near the supply values was expected. However, the actual results are shown in Figs. $9 \& 10$.

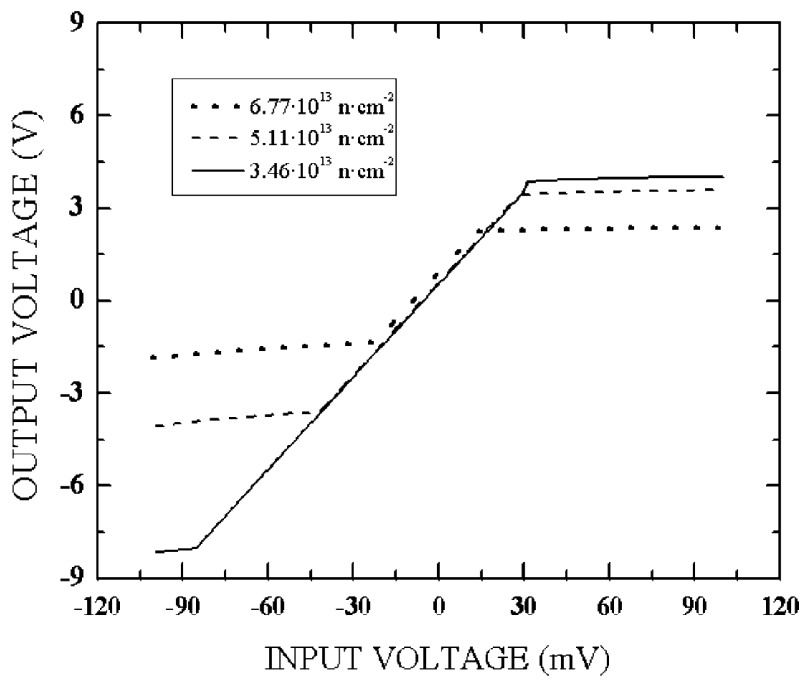

Figure 9. Input-output function of several INA110KP samples. They were irradiated with different doses. Inverting input grounded.

Fig. 9 shows that the saturation voltage value decreases in proportion to the neutron dose received. This phenomenon is much more important in the case of positive saturation voltage. In Fig. 10, we observe that the saturation voltages do not depend on the sign of the gain. Unlike INA110KP, the saturation voltage of INA111BP is not horizontal and the negative saturation voltage is lower than the positive one.

\section{RESULTS ON BUILT AMPLIFIERS}

The instrumentation amplifiers built with OPA627AP and TLE2071CP operational amplifiers received a total neutron dose between $2-7 \cdot 10^{13} \mathrm{n} / \mathrm{cm}^{2}$ without being strongly altered. Only a slight gain reduction and an input offset shift were observed. The shift of the offset voltage is quite similar to that one observed on the operational amplifiers [7]. No change on input bias and offset was measured. The degradation of

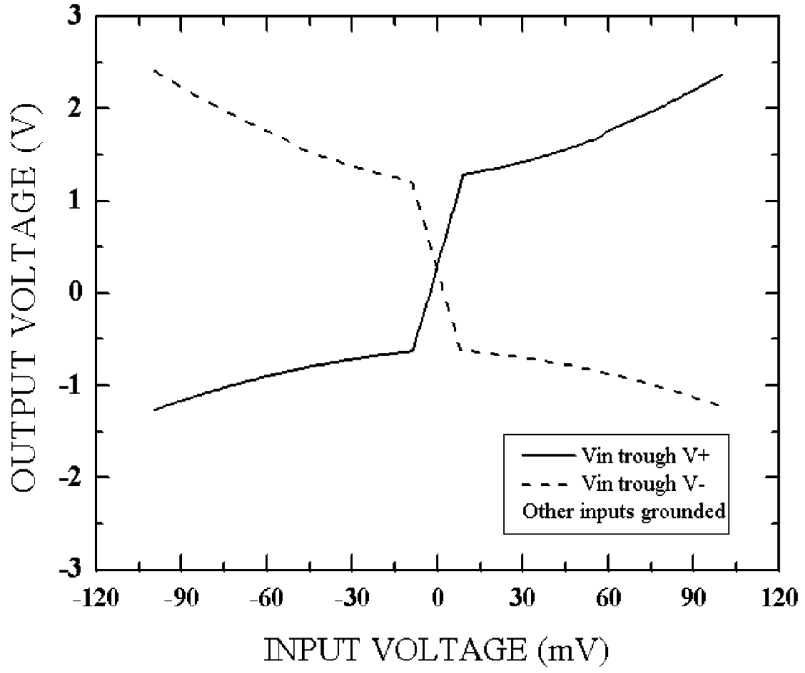

Figure 10. Input-output function of an INA111PA sample that received a total neutron fluence of $5.89 \cdot 10^{13} \mathrm{n} / \mathrm{cm}^{2}$.

their properties is related to the modification of the individual operational amplifiers.

\section{DISCUSSION}

The tested devices were built in bipolar technology with JFET input stage in some of them. Gamma radiation can affect this kind of technology in the same way as neutrons, but the damage is much lower than the one caused by displacement [8], [9]. An additional effect that is produced by ionizing radiation on integrated bipolar devices is the store of positive charge inside the epitaxial oxide. This charge allows the creation of leakage currents and leads to an increase of the input bias currents [10].

\section{A. Differential Gain}

The behavior of the amplifier gain can be explained remembering two effects that happen when a semiconductor is exposed to a radiation that originates NIEL damage: the reduction of the gain of bipolar transistors due to the growing importance of base recombination currents and the semiconductor resistivity growth.

The instrumentation amplifier shown in Fig. 1 can be converted into the network of Fig. 11 replacing the operational amplifier by a dependent voltage source with a very high but not infinite gain. The resolution of a system with seven linear equations leads to the following value of the gain $G=V_{O U T} / V_{I N}$ :

$$
G \cong G_{\infty} \cdot \frac{1+A_{1}^{-1}}{1+\frac{1}{2} \cdot\left(G_{\infty}+1\right) \cdot\left(A_{1}^{-1}+A_{2}^{-1}\right)+2 \cdot A_{3}^{-1}}
$$

$\mathrm{G}_{\infty}$ is the value of the gain of the instrumentation amplifier with ideal operational amplifiers, and its value is $\left(1+2 \mathrm{R} / \mathrm{R}_{G}\right)$. The bipolar transistor gain decreases with neutron radiation [8] as

$$
\beta^{-1}=\beta_{0}^{-1}+K_{\beta} \cdot \Phi
$$

where $K_{\beta}$ is a constant that depends on the semiconductor, the energy of the neutrons, temperature, and so on, and $\Phi$ is the 


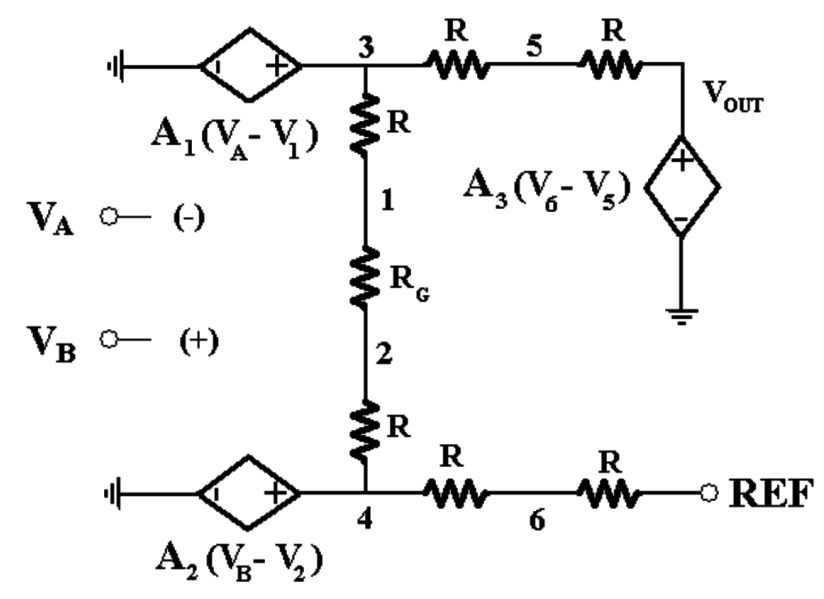

Figure 11. Instrumentation amplifier with opamps as dependent sources.

total neutron fluence. As the open loop gain of operational amplifiers is related to the gain of individual transistors, we conclude that the open loop gain decreases with $\Phi$.

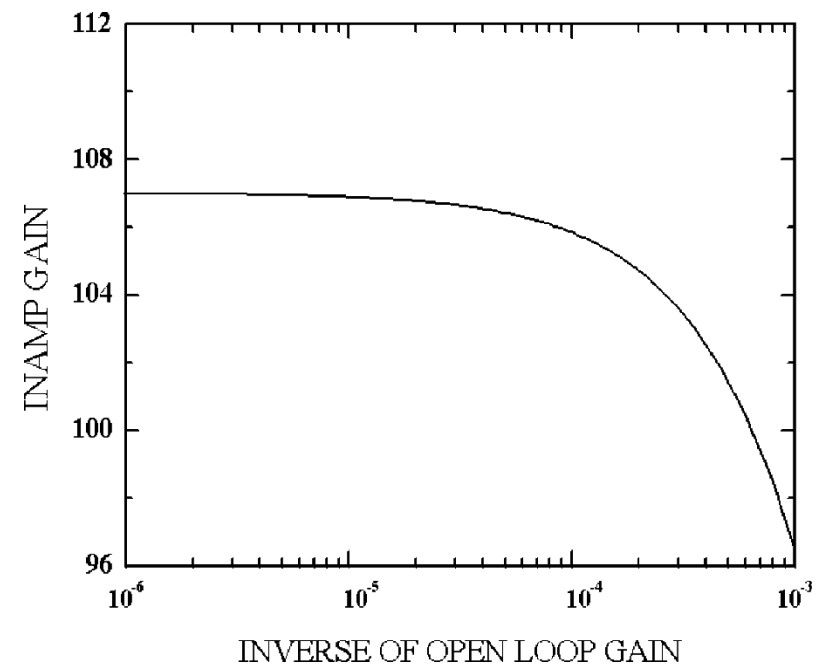

Figure 12. Gain of an instrumentation amplifier depends on the open loop gain of integrated op amps.

The relation of $G$ with the operational amplifier gain has been drawn in Fig. 12. For simplicity, we assumed that $G_{1}=G_{2}=G_{3}=G_{O L}$. So, the reduction of instrumentation amplifiers gain can be related to the decrease of the open loop gain of the integrated operational amplifiers. However, other amplifiers show a gain growth before a sudden drop to zero. This increase can be justified with the growth of the resistivity of semiconductors. The internal resistors of the devices are made with doped silicon and its value grows when they are irradiated. The resistivity of the semiconductor follows the mathematical expression [8]:

$$
\rho=\rho_{0} \cdot \exp \left(\frac{\Phi}{K_{\rho}}\right)
$$

$K_{\rho}$ is a constant that depends on the material, the neutron energy, etc. Due to the increase of resistivity, the internal resistors are higher and, according to 1, the gain must increase because the external resistance $R_{G}$ is not a semiconductor resistor and does not suffer any change. However, we do not reject that other unidentified mechanisms could cause the differential gain increase. On the other hand, the difference among the internal resistances, very similar but not completely matched, grows exponentially to the neutron fluence and can lead to more complex evolutions than the one deduced from the combination of $1 \& 4$.

Finally, there are mechanisms that modify the gain in two different ways. Generally, the competition among different mechanisms on irradiated electronic devices has been described in the related literature [11], [12], [13].

\section{B. Input Offset Voltage}

Another interesting item is the different evolution of input offset voltage if the technology is bipolar or JFET. The appearance of offset voltage in any differential device is due to the asymmetry of the building of the network parts, mainly on the input stage [14], [15]. The matching among devices is much more difficult in JFET technology, and generally, the amplifiers with JFET input have a higher offset voltage than the bipolar ones [16], [17]. The great reproducibility in bipolar technology allows to build similar input stages in the samples of the same batch. Therefore, the input offset voltage of bipolar instrumentation must behave in a similar way if they are irradiated. On the other hand, the input stage of JFET amplifiers changes from sample to sample. Due to the intrinsic difference, the evolution is random because it depends strongly on the manufacturing conditions of the sample.

\section{Bias Input Currents and Frequency Behavior}

The growth of bias current is due to two reasons. First, the increase of leakage currents in the PN junction that makes both the bipolar or JFET input because they depend on the minority carrier lifetime [8], [18]. On the other hand, leakage currents appear because of the appearance of charged particles inside the epitaxial oxide. Both processes have the same consequence [19], [20], and we do not know which is the main cause. The worsening of frequency response is directly related to the decrease of the minority carrier lifetime [8] since this is the main parameter that controls the frequency behavior of bipolar devices.

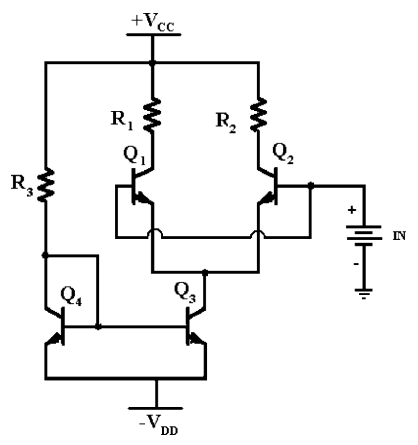

Figure 13. Differential amplifier. 


\section{Quiescent Current}

The drop of quiescent current values is a consequence of the alteration of the internal device components. Fig. 13 shows the simplest differential amplifier. Operational amplifiers are built with several differential amplifiers. The quiescent current of this amplifier is determined by $R_{3}$ and the current mirror built with $\mathrm{Q}_{3} \& \mathrm{Q}_{4}$. We suppose that the transistors are similar so $\beta_{3}=\beta_{4}=\beta$. On the other hand, when a transistor is irradiated, base recombination currents appear and

$$
\begin{gathered}
I_{B}=I_{B 1}+I_{R} \\
I_{C}=\beta \cdot I_{B 1}
\end{gathered}
$$

$I_{B}$ is the current that goes into the transistor base, $I_{R}$ the recombination current and $I_{C}$ the collector current. The resolution of the network equation gives the following value of current required by the amplifier:

$$
I_{Q}=\left(1+\frac{\beta}{(\beta+2)+2 \cdot \frac{I_{R}}{I_{B 1}}}\right) \cdot \frac{V_{C C}+V_{D D}-V_{B E(O N)}}{R_{3}}
$$

We have accepted that the recombination currents are similar in both bipolar transistors. This current decreases if $\mathrm{R}_{3}$ or $\mathrm{I}_{R}$ grow, and this happens when a device is irradiated. $V_{B E(O N)}$ can change, but its influence in $\mathrm{I}_{Q}$ is negligible since it is added to $V_{C C}+V_{D D} \sim 30 \mathrm{~V}$. Other current mirrors as base current compensated, cascode or Wilson have the same behavior when the recombination current grows. Therefore, a decrease of the current required by the differential amplifiers is predicted so the quiescent current of instrumentation amplifiers falls. The measured values shown in Fig. 8 confirm the result. This phenomenon is opposed to the evolution of consumption in integrated CMOS devices where a growth of supply current is expected due to the storage of charged particles inside the epitaxial oxide [9], [10], [11]. Moreover, in low power design, the use of low gain transistors and high resistors is advisable to reduce the currents. So, the action of displacement damage is more important than in the other amplifiers.

\section{E. Saturation Voltages}

Finally, the reason for the decrease of the device saturation voltage is interesting. First of all, the shift could be attributed to the modification of saturation voltage of individual operational amplifiers. However, the data of the irradiated operational amplifiers that were found in the Internet databases [21], [22] do not support this theory since the shifts are in the order of one or two tenths of volts. No amplifier shows a saturation voltage swing as great as the one observed on the instrumentation amplifiers. Our proposal is the following: The integrated operational amplifiers are loaded with resistors about $25 \mathrm{k} \Omega$. One of the most affected parameters in operational amplifiers is the short circuit current. This is the highest current that an electronic device can supply. The results of an operational amplifier OP-11, found at ERRIC database, show that the positive short circuit current falls from 9 to $2.51 \mathrm{~mA}$ and the negative one from 10.6 to $1.74 \mathrm{~mA}$ when it was exposed up to $10^{13} \mathrm{n} / \mathrm{cm}^{2}$. This event is related to the degradation of the output stage of the operational amplifiers; e.g., the push-pull output stage like Fig. 14 is commonly used to build integrated operational amplifiers [23]. The highest positive current supplied by this network is proportional to $h_{F E, 1}$. The value of this parameter gets low due to the appearance of recombination current, so the positive output current decreases. A similar result is obtained in the negative output current, but it depends on $h_{F E, 2}$. Moreover, if the degradation of the transistors $Q_{1} \& Q_{2}$ is not similar, the evolution of the short circuit current depends on their sign. So, the results shown in Figs. 9 and 10 are caused by the incapacity of operational amplifiers to supply enough output current and bias the resistance network of Fig. 1. In addition, when the output of the instrumentation amplifier is loaded, the saturation voltage is lower than the values given in Figs. 9 and 10.

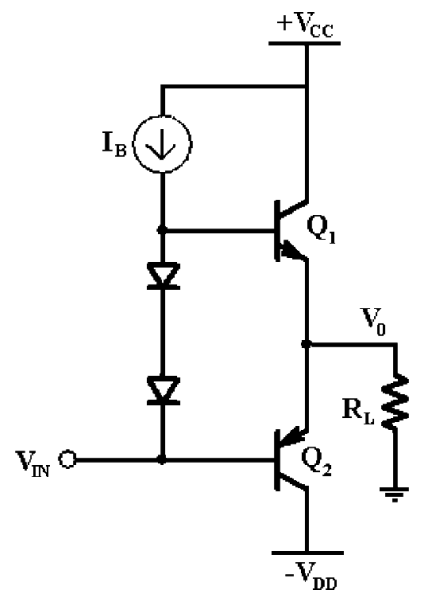

Figure 14. Push-pull output stage.

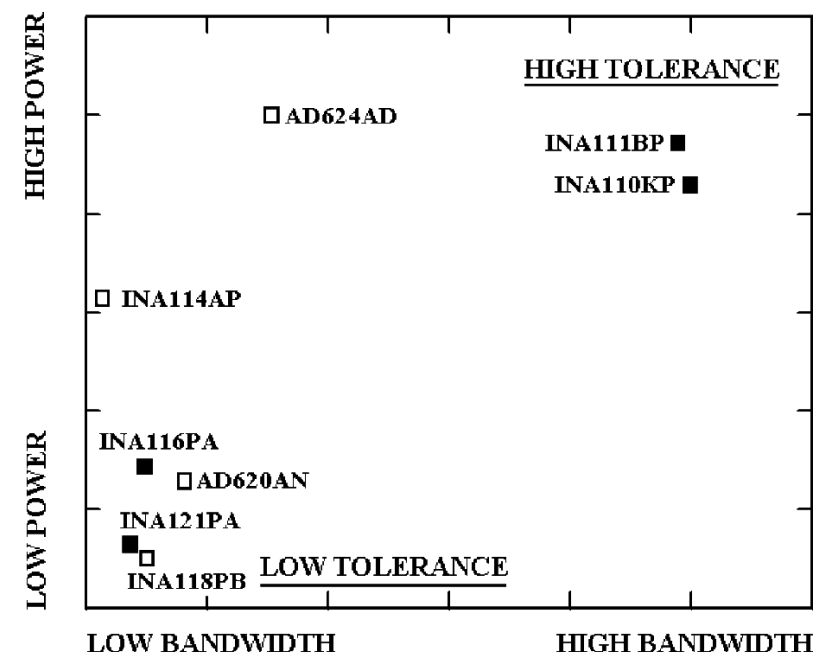

Figure 15. NIEL tolerance of instrumentation amplifiers. The bipolar input devices are symbolized with empty squares.

\section{F. Built Instrumentation Amplifiers}

Finally, the alternative solution of building instrumentation amplifiers with operational amplifiers has the advantage of a good radiation tolerance, which is higher than in the integrated 
commercial amplifiers. However, the great problem of tuning remains. It is very difficult to obtain a high CMRR value with discrete components. This has been studied at [24], where other disadvantages are enumerated: higher temperature influence, nonlinearity, and even a cost increase.

\section{CONCLUSION}

The development of the instrumentation that has to be exposed to neutron radiation can require the use of instrumentation amplifiers. It has been shown that broad bandwidth amplifiers present a high radiation tolerance, that low-power design decreases the rad-tolerance, and that the JFET input amplifiers are more tolerant than the completely bipolar amplifiers. Fig. 15 shows their predicted radiation tolerance. The $\mathrm{X}$ axis is the frequency response, computed from $-3 \mathrm{~dB}$ frequency and slew rate, and the $\mathrm{Y}$-axis is the power consumption in arbitrary units. The distance to the origin allows to estimate the tolerance of the devices.

The bipolar input amplifiers show the advantage of a similar evolution of input offset voltage in all of the devices of the same manufacturer batch. However, the highest input bias currents were found on amplifiers of this technology. The output saturation voltage will be lower, and the consumption is going to decrease.

The building of instrumentation amplifiers is one possible option, but the response against temperature, common mode voltage, etc. is not as good as that of integrated commercial amplifiers.

\section{REFERENCES}

[1] The LHC Study Group, "The Large Hadron Collider Conceptual Design,” Tech. Rep., 1995.

[2] J. R. Riskin, "A User's Guide to IC Instrumentation Amplifier ," [Online] Available: Appl. Notes Analog Devices AN-244; Analog Devices website.

[3] C. Kitchin and L. Counts, "A Designer's Guide to Instrumentation Amplifiers," [Online] Available: Appl. Guide Analog Devices; Analog Devices website, Tech. Rep., 2000.

[4] P. Horowitz and W. Hill, The Art of Electronics, 2nd ed. Cambridge Univ. Press, 1990

[5] "Op Amp Circuit Collection," [Online] Available: Appl. Note National Semiconductor AN-31; National Semiconductor website, Oct. 2000.

[6] J. A. Agapito et al., "Instrumentation amplifiers and voltage controlled current sources for LHC cryogenic system," in 6th Workshop LHC Experiments (LEB'O0), Sep. 2000, pp. 275-280.

[7] — "Preliminary test for radiation tolerant electronic components for the LHC cryogenic system," in 5th Workshop LHC Experiments (LEB'99), Sep. 1999, pp. 475-479.

[8] G. Messenger and M. Ash, The Effects of Radiation on Electronic Systems, 2nd ed. Van Nostrand Reinhold, 1992.

[9] L. Adams, "Guidelines for the Use of Electronic Components in the Space Radiation Environment," Tech. Rep., 2000.

[10] F. Faccio, "COTS for LHC radiation environment: The rules of the game," in 6th Workshop LHC Experiments (LEB'O0), Sep. 2000, pp. $50-63$.

[11] H. J. Barnaby et al., "Identification of degradation mechanisms in a bipolar linear voltage comparator trough correlation of transistor and circuit response," IEEE Trans. Nucl. Sci., vol. 47, pp. 1666-1673, Dec. 1999.

[12] — "Origins of total dose response variability in linear bipolar microcircuits," IEEE Trans. Nucl. Sci., vol. 46, pp. 2342-2349, Dec. 1999.

[13] F. Saigné et al., "Experimental procedure to predict the competition between the degradation induced by irradiation and thermal annealing of oxide trapped charge in MOSFETs," IEEE Trans. Nucl. Sci., vol. 47, pp. 2329-2333, Dec. 2000.
[14] D. Christiansen, Electronics Engineers' Handbook, 4th ed. McGrawHill, 1997.

[15] E. Greeneich, Analog Integrated Circuits, London, U.K.: Chapman Hall, 1997.

[16] R. Palmer, "DC parameters: Input offset voltage (VIO)," [Online] Available: Appl. Note Texas Instruments; Texas Instruments website, Mar. 2001.

[17] B. Ngouyen and W. D. Smith, "Nulling input offset voltage of operational amplifiers," [Online] Available: Appl. Note Texas Instruments; Texas Instruments website, Aug. 2000.

[18] D. Neamen, Semiconductor Physics and Devices: Basic Principles, 2nd ed. McGraw-Hill, 1992.

[19] B. G. Rax, A. H. Johnston, and T. Miyahira, "Displacement damage in bipolar linear integrated circuits," IEEE Trans. Nucl. Sci., vol. 46, pp. 1660-1665, Dec. 1999.

[20] H. J. Barnaby, R. D. Schrimpf, R. D. Sternberg, V. Berthe, C. R. Cirba, and R. L. Pease, "Proton radiation response mechanisms in bipolar analog circuits," IEEE Trans. Nucl. Sci., vol. 48, pp. 2074-2080, Dec. 2001.

[21] [Online]. Available: http://erric.dasiac.com

[22] "Jet Propulsion Laboratory Radata Interactive ," [Online]. Available: http://radnet.jpl.nasa.gov.

[23] W. K. Chien, The VLSI Handbook. CRC Press, 2000.

[24] E. Nash, "Errors and error budget analysis in instrumentation amplifiers applications," [Online] Available: Appl. Note from Analog Devices; Analog Devices website. 